\title{
DGN-Jahresberichte 2018
}

Die folgenden Tabellen stellen die Aktivitäten der DGN-Ausschüsse ( $\triangleright$ Tab. 1) und Arbeitsgemeinschaften ( $\triangleright$ Tab. 2) sowie der Regionalgesellschaften ( $\triangleright$ Tab. 3) im Berichtszeitraum 2018 dar.

D Tab. 1 Jahresberichte 2018: DGN-Ausschüsse

\begin{tabular}{|c|c|c|}
\hline Ausschuss, & \multicolumn{2}{|c|}{ Zusammenkünfte, Aktivitäten im Berichtszeitraum und Planungen } \\
\hline \multirow[t]{2}{*}{$\begin{array}{l}\text { Dosimetrie, } \\
\text { Dr. M. Hohberg }\end{array}$} & Veranstaltungen & $\begin{array}{l}\text { - Sitzung im Rahmen der DGN-Jahrestagung, 19.04.2018, Bremen } \\
\text { - Telefonkonferenz, 29.11.2018 }\end{array}$ \\
\hline & Vorhaben & $\begin{array}{l}\text { - Erstellung eines elektronischen Fragebogens um einen Überblick zu erhalten wie in Deutschland Therapie, } \\
\text { Therapieplanung, Bildgebung und Dosimetrie durchgeführt werden } \\
\text { - Zusammenarbeit mit DGN-Ausschüssen Medizinphysik und Therapie }\end{array}$ \\
\hline \multirow[t]{3}{*}{$\begin{array}{l}\text { Ethik, } \\
\text { Prof. Dr. } \\
\text { W. H. Knapp }\end{array}$} & Veranstaltungen & $\begin{array}{l}\text { - Sitzung im Rahmen der DGN-Jahrestagung, 20.04.2018, Bremen } \\
\text { - Ausschuss-Sitzung, 11.07.2018, Hannover } \\
\text { - Beratung mit Geschäftsstelle, 14.09.2018, Göttingen }\end{array}$ \\
\hline & Aktivitäten & $\begin{array}{l}\text { - Erstellung von „Grundsätzen zu Transparenz, Ethik und Vermeidung von Interessenkonflikten“, einer Geschäfts- } \\
\text { ordnung und von Verfahrensstandards zur Bewertung von Sekundärinteressen } \\
\text { - Diverse Abstimmungen fernmündlich und elektronisch } \\
\text { - Implementierung der Erklärung sekundärer Interessen in elektronischer Form } \\
\text { - Zusammenarbeit mit DGN-Gremien } \\
\text { DGN-Vorstand Finalisierung der Verfahrensstandards etc., 18.11.2018 } \\
\text { - Anderweitige Aktivitäten (z. B. Studien, Publikationen) } \\
\text { Internetauftritt des Ausschusses Ethik }\end{array}$ \\
\hline & Vorhaben & - Bewertung der Erklärungen von Sekundärinteressen mit Empfehlungen an den Vorstand, bis Frühjahr 2019 \\
\hline \multirow{3}{*}{$\begin{array}{l}\text { Fort- und } \\
\text { Weiterbildung, } \\
\text { Prof. Dr. W. } \\
\text { Brenner }\end{array}$} & Veranstaltungen & $\begin{array}{l}\text { - Sitzung im Rahmen der DGN-Jahrestagung 2018,18.04.2018, Bremen } \\
\text { - Ausschuss-Sitzung, 16.11.2018, Berlin } \\
\text { - Monatliche Telefonkonferenzen }\end{array}$ \\
\hline & Aktivitäten & $\begin{array}{l}\text { - Fort- und Weiterbildungsaktivitäten } \\
\text { - Organisation CME-Sessions der Jahrestagungen } 2018 \text { und } 2019 \\
\text { - Beteiligung an DGN-Veranstaltungen oder anderen Veranstaltungen } \\
\text { - DGN Summer School: „Translational Research in Molecular Imaging and Radionuclide Therapy“, 30.08.- } \\
\text { 01.09.2018, Berlin } \\
\text { - Vorbereitung der DGN Summer School } 2019 \\
\text { - Vorbereitung der CME-Sessions der DGN-Jahrestagung } 2019 \\
\text { - Teilnahme UEMS/EBNM National Delegates Assembly während der EANM } 2018 \text { in Düsseldorf (M. Gotthardt) } \\
\text { - Zusammenarbeit mit DGN-Gremien } \\
\text { - Konzepterstellung einer „Akademie Nuklearmedizin“ mit dem Vorstand } \\
\text { - Zusammenarbeit mit anderen Gremien/Kommissionen } \\
\text { - CME-Sessions der DGN-Jahrestagung je nach Thema in Abstimmung mit dem jeweils zuständigen Ausschuss } \\
\text { - Anderweitige Aktivitäten (z. B. Studien, Publikationen) } \\
\text { - Organisation Mitarbeit Pschyrembel: Fachbereich Nuklearmedizin und zugehörige Physik, Medizintechnik und } \\
\text { Radiopharmazie für Printausgabe 2018 sowie für Online-Ausgabe 2019 } \\
\text { - Publikation: Profes. J. Marienhagen, W. Brenner, A. Buck, C. Franzius, LS. Freudenberg, M. Gotthardt, O. Lind- } \\
\text { ner, F. M. Mottaghy, D. Hellwig Development of a national competency-based learning objective catalogue for } \\
\text { undergraduate medical education in Germany. Nuklearmedizin. 57:137-145 (2018) }\end{array}$ \\
\hline & Vorhaben & $\begin{array}{l}\text { - DGN-Summer School 2019, 29.-31.08.2019, Berlin } \\
\text { - Ausschuss-Sitzungen im Rahmen der DGN-Jahrestagung } 2019 \text { und Ausschuss-Sitzung im November in Berlin } \\
\text { - Pschyrembel Online-Edition, 1. Halbjahr } 2019 \\
\text { - Begleitung Musterweiterbildungsordnung } \\
\text { - Prüfung Antragstellung BMBF-Bekanntmachung der 3. Förderlinie „Disziplin- und fachbezogene digitale Hoch- } \\
\text { schulbildung“ im Rahmen der Projektträgerschaft Digitale Hochschulbildung } \\
\text { - Paper zum Thema „Statuserhebung der Studentischen Lehre für das Fach Nuklearmedizin in Deutschland“ in } \\
2019\end{array}$ \\
\hline
\end{tabular}


- Tab. 1 Fortsetzung

\begin{tabular}{|c|c|c|}
\hline Ausschuss, & \multicolumn{2}{|c|}{ Zusammenkünfte, Aktivitäten im Berichtszeitraum und Planungen } \\
\hline \multirow{3}{*}{$\begin{array}{l}\text { Leistungs- } \\
\text { erfassung und } \\
\text {-vergütung, } \\
\text { PD Dr. M. } \\
\text { Freesmeyer }\end{array}$} & Veranstaltungen & $\begin{array}{l}\text { - Telefonkonferenz, } 11.01 .2018 \\
\text { - Sitzung im Rahmen der DGN-Jahrestagung, 18.04.2018, Bremen } \\
\text { - Telefonkonferenz, 26.09.2018 } \\
\text { - Ausschuss-Sitzung, 16.11.2018 }\end{array}$ \\
\hline & Aktivitäten & $\begin{array}{l}\text { - Fort- und Weiterbildungsaktivitäten } \\
\text { - Evaluation einer Fortbildungsveranstaltung „Kodierworkshop“ } \\
\text { - Update DRG (Dr. W. Fiori) auf der Jahrestagung DGN 2018, Bremen } \\
\text { - Zusammenarbeit mit DGN-Gremien } \\
\text { - Ausschuss Fort- und Weiterbildung } \\
\text { - Zusammenarbeit mit anderen Fachgesellschaften } \\
\text { - Deutsche Gesellschaft für Urologie } \\
\text { - Anderweitige Aktivitäten (z. B. Studien, Publikationen) } \\
\text { - Erfolgreiche Beantragung eines eigenständigen OPS „Intratherapeutische Dosimetrie“ 8-539.0 } \\
\text { - Erfolgreiche Beantragung einer Änderung des Hinweistextes OPS 8-530 } \\
\text { - Information in eBrief zu NUB-Antrag Lu-PSMA // DRG-Inklusion ab 2019 } \\
\text { - Erstellung eines Fragebogens, der deutschlandweit Zusatz- und NUB-Entgelte erfasst (Distribution Anfang 2019) } \\
\text { - Neuerstellung der Kodierempfehlungen gemäß des DRG- und OPS-Katalogs } 2019 \text { sowie Distribution an } \\
\text { die deutschlandweiten Kalkulationskrankenhäuser via Geschäftsstelle mit dem Ziel der einheitlichen und } \\
\text { korrekten Kodierung } \\
\text { - Vorbereitung OPS-Änderungen } \\
\text { - 8-530.60 (Abrechnung Lutathera) } \\
\text { - 8-530.60/3-754 (Öffnung für weitere Chelatoren außer DOTA) } \\
\text { - 3-70c.3 (Tumorszintigraphie mit Tc-PSMA) in Verbindung mit Radioguided Surgery } \\
\text { - 3-709.0 (Tilmanocept) }\end{array}$ \\
\hline & Vorhaben & $\begin{array}{l}\text { - Beantragung o.g. OPS-Änderungen } \\
\text { - Aktive Prüfung des Sachkostenanteils M10B } \\
\text { - (Lu-PSMA-Therapie), Erarbeitung einer spezifischen Kodierempfehlung und Distribution an die Kalkulations- } \\
\text { krankenhäuser, um eine Abwertung } 2020 \text { zu verhindern } \\
\text { - Auswertung des Fragebogens, der Zusatz- und NUB-Entgelte erfasst } \\
\text { - Überarbeitung und Veröffentlichung der Kodierrichtlinien für } 2020 \\
\text { - Erarbeitung einer OPS-Änderung } 8-530 . d 0 \text { analog } 8-530.60, \text { wenn zugelassenes Lu-PSMA zur Verfügung steht }\end{array}$ \\
\hline \multirow[t]{2}{*}{$\begin{array}{l}\text { Leitlinien, } \\
\text { Prof. Dr. M. } \\
\text { Schmidt }\end{array}$} & Veranstaltungen & $\begin{array}{l}\text { - Sitzung im Rahmen der DGN-Jahrestagung, 18.04.2018, Bremen } \\
\text { - Ausschuss-Sitzung, 16.11.2018, Berlin } \\
\text { - S3-Leitlinienprojekt „Schilddrüsenkarzinom“, Treffen der federführenden Fachgesellschaften, 20.06.2018, Berlin } \\
\text { und Kick-off-Treffen, 22.11.2018, Essen }\end{array}$ \\
\hline & Aktivitäten & $\begin{array}{l}\text { - Leitlinien Publikationen in 2018: } \\
\text { - Somatostatinrezeptor-PET/CT } \\
\text { - Lungenszintigraphie Version } 3 \\
\text { - Leitlinien: Mitwirkung an Nationalen Versorgungsleitlinien } \\
\text { - Chronische KHK } \\
\text { - Mitwirkung an Leitlinien des Leitlinienprogramms Onkologie } \\
\text { - Analkarzinom } \\
\text { - Chronisch lymphatische Leukämie } \\
\text { - Endometriumkarzinom } \\
\text { - Follikuläres Lymphom } \\
\text { - Hepatocelluläres Karzinom } \\
\text { - Hodenkarzinom } \\
\text { - Hodgkin-Lymphom } \\
\text { - Larynxkarzinom } \\
\text { - Lungenkarzinom } \\
\text { - Magenkarzinom } \\
\text { - Monoklonale Gammopathie unklarer Signifikanz (MGUS) oder Multiplem Myelom } \\
\text { - Melanom } \\
\text { - Mundhöhlenkarzinom } \\
\text { - Nierenzellkarzinom } \\
\text { - Ovarialkarzinom } \\
\text { - Ösophaguskarzinom } \\
\text { - Pankreaskarzinom } \\
\text { - Peniskarzinom } \\
\text { - Prostatakarzinom } \\
\text { - Zervixkarzinom }\end{array}$ \\
\hline
\end{tabular}


- Tab. 1 Fortsetzung

\begin{tabular}{|c|c|c|}
\hline Ausschuss, & \multicolumn{2}{|c|}{ Zusammenkünfte, Aktivitäten im Berichtszeitraum und Planungen } \\
\hline $\begin{array}{l}\text { Leitlinien } \\
\text { (Fortsetzung) }\end{array}$ & $\begin{array}{l}\text { Aktivitäten } \\
\text { (Fortsetzung) }\end{array}$ & 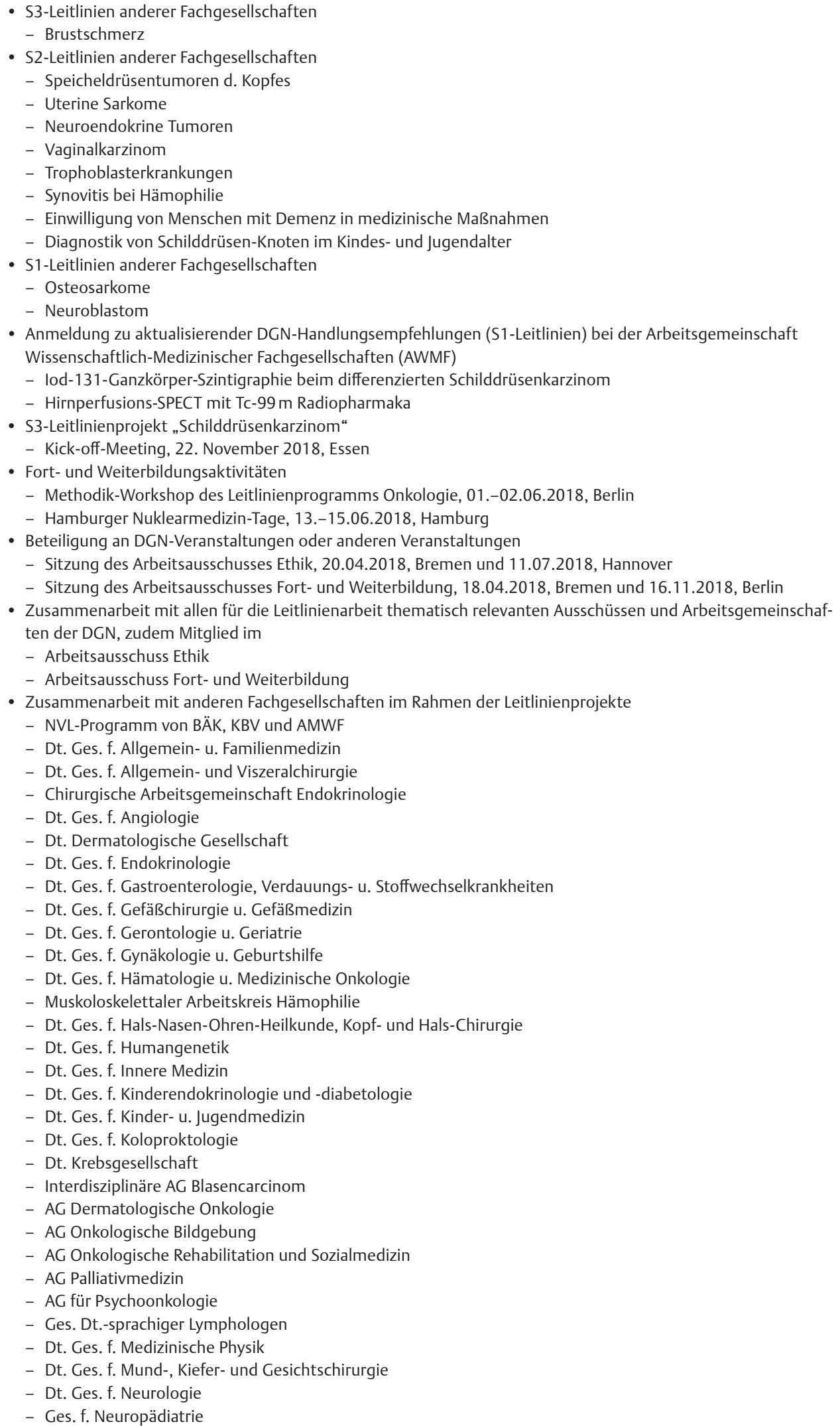 \\
\hline
\end{tabular}


- Tab. 1 Fortsetzung

\begin{tabular}{|c|c|c|}
\hline $\begin{array}{l}\text { Ausschuss, } \\
\text { Vorsitz }\end{array}$ & \multicolumn{2}{|c|}{ Zusammenkünfte, Aktivitäten im Berichtszeitraum und Planungen } \\
\hline \multirow[t]{2}{*}{$\begin{array}{l}\text { Leitlinien } \\
\text { (Fortsetzung) }\end{array}$} & $\begin{array}{l}\text { Aktivitäten } \\
\text { (Fortsetzung) }\end{array}$ & $\begin{array}{l}\text { - Zusammenarbeit mit anderen Fachgesellschaften im Rahmen der Leitlinienprojekte (Fortsetzung) } \\
\text { - Ges. f. Pädiatrische Onkologie u. Hämatologie } \\
\text { - Dt. Ges. f. Palliativmedizin } \\
\text { - Dt. Ges. f. Pathologie } \\
\text { - Dt. Ges. f. Phoniatrie und Pädaudiologie } \\
\text { - Dt. Ges. f. Plastische u. Wiederherstellungschirurgie } \\
\text { - Dt. Ges. f. Pneumologie u. Beatmungsmedizin } \\
\text { - Dt. Ges. f. Psychiatrie und Psychotherapie, Psychosomatik u. Nervenheilkunde } \\
\text { - Dt. Ges. f. Radioonkologie } \\
\text { - Dt. Ges. f. Rehabilitationswissenschaften } \\
\text { - Dt. Röntgengesellschaft } \\
\text { - Dt. Ges. f. Ultraschall in der Medizin } \\
\text { - Dt. Ges. f. Urologie } \\
\text { - Fachverband für Strahlenschutz } \\
\text { - Zusammenarbeit mit Patientenvertretungen } \\
\text { - Bundesverband Schilddrüsenkrebs - Ohne Schilddrüse leben } \\
\text { - Schilddrüsen-Liga Deutschland } \\
\text { - Zusammenarbeit mit anderen Gremien/Kommissionen } \\
\text { - Treffen mit Vertretern der Deutschen Röntgengesellschaft zur Zusammenarbeit von Radiologie und Nuklear- } \\
\text { medizin bei der S3-LL-Aktualisierung HCC / CCC, 18.-19.10.2018, München } \\
\text { - Zusammenarbeit mit Patientenvertretungen } \\
\text { - Bundesverband Schilddrüsenkrebs - Ohne Schilddrüse leben } \\
\text { - Schilddrüsen-Liga Deutschland } \\
\text { - Anderweitige Aktivitäten } \\
\text { - Publikation von DGN-Handlungsempfehlungen (S1-Leitlinien) im Fachjournal „Nuklearmedizin“ } \\
\text { - Somatostatinrezeptor-PET/CT (Ausgabe 1/2018) } \\
\text { - Lungenszintigraphie (Ausgabe 3/2018) } \\
\text { - lod-131-Ganzkörperszintigraphie beim differenzierten Schilddrüsenkarzinom (Ausgabe 4/2018) }\end{array}$ \\
\hline & Vorhaben & S3-Leitlinie Schilddrüsenkarzinom, Beginn der Erstellung der Leitlinien-Texte in 2019 \\
\hline \multirow[t]{3}{*}{$\begin{array}{l}\text { Medizinphysik, } \\
\text { PD Dr. S. Nekolla }\end{array}$} & Veranstaltungen & $\begin{array}{l}\text { - Sitzung im Rahmen der DGN-Jahrestagung, 18.04.2018, Bremen } \\
\text { - Telefonkonferenz, 20.12.2018 }\end{array}$ \\
\hline & Aktivitäten & $\begin{array}{l}\text { - Beteiligung an DGN-Veranstaltungen oder anderen Veranstaltungen } \\
\text { - GAPTN } \\
\text { - Zusammenarbeit mit DGN-Gremien } \\
\text { - Ausschuss für Dosimetrie } \\
\text { - Ausschuss Strahlenschutz } \\
\text { - Ausschuss Therapie } \\
\text { - Zusammenarbeit mit anderen Fachgesellschaften } \\
\text { - EANM Dosimetry Committee } \\
\text { - EANM Physics Committee } \\
\text { - EANM Radiation Protection Committee } \\
\text { - DGMP (GAPTN) }\end{array}$ \\
\hline & Vorhaben & $\begin{array}{l}\text { - Aufbau eines CME-Curriculums „Quantifizierung“ im Rahmen von Refresherkursen während der DGN-Jahres- } \\
\text { tagung. Dieses Curriculum ist auf vier Jahre angelegt und beinhaltet die Module } \\
\text { 1. Quantifizierung in der nuklearmedizinischen Bildgebung I: Messtechnische Aspekte, Korrekturen und } \\
\text { quantitative Größen } \\
\text { 2. Quantifizierung in der Therapie I: Grundlagen } \\
\text { 3. Quantifizierung in der nuklearmedizinischen Bildgebung II: Neue Methoden und Qualitätskontrolle } \\
\text { 4. Quantifizierung in der Therapie II: Update der Grundlagen und spezielle Aspekte, Ab DGN-Jahrestagung } 2019 \\
\text { - Aufbau einer Infrastruktur für eine web-basierte Artefakt-Datenbank durch die Klinik für Nuklearmedizin der Uni- } \\
\text { versität Leipzig (technische Unterstützung weiterhin erforderlich - derzeit Testphase auf proprietärer Infrastruk- } \\
\text { tur, perspektivisch Providing durch DGN/vokativ), Evaluierung durch Ausschussmitglieder in 2019. Vorstellung } \\
\text { des Status auf DGMP-Winterschule in Pichl (03/2019), dann Erweiterung des Kreises der Testbenutzer }\end{array}$ \\
\hline \multirow{2}{*}{$\begin{array}{l}\text { Multicenter- } \\
\text { Studien, } \\
\text { Prof. Dr. C. la } \\
\text { Fougère }\end{array}$} & Veranstaltungen & $\begin{array}{l}\text { - Sitzung im Rahmen der DGN-Jahrestagung, 18.04.2018, Bremen } \\
\text { - Ausschuss-Sitzung, 16.11.2018, Berlin }\end{array}$ \\
\hline & Aktivitäten & $\begin{array}{l}\text { - Zusammenarbeit mit DGN-Gremien } \\
\text { - Ausschuss Radiopharmazie } \\
\text { - Anderweitige Aktivitäten (z. B. Studien, Publikationen) } \\
\text { - Vorstellung der Ergebnisse des Ausschusses an DGN-Vorstand }\end{array}$ \\
\hline
\end{tabular}


- Tab. 1 Fortsetzung

\begin{tabular}{|c|c|c|}
\hline $\begin{array}{l}\text { Ausschuss, } \\
\text { Vorsitz }\end{array}$ & \multicolumn{2}{|c|}{ Zusammenkünfte, Aktivitäten im Berichtszeitraum und Planungen } \\
\hline \multirow{3}{*}{$\begin{array}{l}\text { PET, } \\
\text { Prof. Dr. H. } \\
\text { Hellwig }\end{array}$} & Veranstaltungen & - Sitzung im Rahmen der DGN-Jahrestagung, 18.04.2018, Bremen \\
\hline & Aktivitäten & $\begin{array}{l}\text { - Leitlinien } \\
\text { - S3 Hodgkin-Lymphom } \\
\text { - S3 Follikuläres Lymphom } \\
\text { - Beteiligung an DGN-Veranstaltungen oder anderen Veranstaltungen } \\
\text { - Theragnostik-Fortbildung auf Deutschem Krebskongress in Berlin } \\
\text { - Eigene Veranstaltungen } \\
\text { - Prüfungen für PET-Zertifikate bei DGN-Jahrestagung } \\
\text { - Zusammenarbeit mit DGN-Gremien } \\
\text { - Ausschuss Strahlenschutz } \\
\text { - Ausschuss Fort- und Weiterbildung } \\
\text { - Ausschuss Leistungserfassung } \\
\text { - Vorstand DGN } \\
\text { - Zusammenarbeit mit anderen Fachgesellschaften } \\
\text { - DRG und mit weiteren im Zusammenhang mit Stellungnahmen an G-BA } \\
\text { - Zusammenarbeit mit anderen Gremien/Kommissionen } \\
\text { - Stellungnahmen zu Beschlussentwürfen des G-BA } \\
\text { - Anderweitige Aktivitäten (z.B. Studien, Publikationen) } \\
\text { - Leitlinie PET/CT } \\
\text { - GOÄ-Reform (Neue Legendierung) } \\
\text { - Prüfung der Anträge auf PET-Zertifikate (DGN-Geschäftsstelle) } \\
\text { - Umfrage zum Indikationsspektrum von PET-Untersuchungen } \\
\text { - Beantwortung von Anfragen bzgl. PET an den „Expertenmakler“ auf der DGN-Internetseite }\end{array}$ \\
\hline & Vorhaben & $\begin{array}{l}\text { - Leitlinie PET/CT 1. Quartal } 2019 \\
\text { - Aktualisierung der Abfrage zu PET/CT-Indikationen in Deutschland } \\
\text { - Prüfungen für PET-Zertifikate während DGN-Jahrestagung 04/2019 }\end{array}$ \\
\hline \multirow[t]{2}{*}{$\begin{array}{l}\text { Radiopharmaka } \\
\text { Prof. Dr. M. Patt }\end{array}$} & Veranstaltungen & $\begin{array}{l}\text { - Sitzung im Rahmen der DGN-Jahrestagung, 18.04.2018, Bremen } \\
\text { - Ausschuss-Sitzung AGRR-Jahrestagung, 21.09.2018, Aachen }\end{array}$ \\
\hline & Aktivitäten & $\begin{array}{l}\text { - Fort- und Weiterbildungsaktivitäten } \\
\text { - GMP-Weiterbildung im Vorfeld der AGRR-Tagung, 20.-22.09.2018, Aachen } \\
\text { Referenten/Thema: } \\
\text { - PhOR R. Schulze, Landesdirektion Sachsen: “Datenintegrität“ } \\
\text { - S. Gärtner, Labor LS } \\
\text { Arbeitsgemeinschaft: „Radiopharmaka-Prüfung auf Sterilität und Endotoxine“ } \\
\text { - Beteiligung an DGN-Veranstaltungen oder anderen Veranstaltungen } \\
\text { - DGN-Jahrestagung } \\
\text { - Eigene Veranstaltungen } \\
\text { - GMP-Schulung im Vorfeld der AGRR-Tagung, 20.-22.09.2018 in Aachen; s. o. } \\
\text { - Zusammenarbeit mit DGN-Gremien } \\
\text { - DGN-Vorstand und Geschäftsstelle } \\
\text { - AGRR } \\
\text { - Telefonkonferenzen } \\
\text { - Zusammenarbeit mit anderen Fachgesellschaften } \\
\text { - EANM } \\
\text { - ISRS } \\
\text { - ESRR } \\
\text { - SNMMI } \\
\text { - Zusammenarbeit mit anderen Gremien/Kommissionen } \\
\text { - EFG12 ZLG } \\
\text { - Arzneibuchkommission BfArM } \\
\text { - Radiopharmacy Committee EANM } \\
\text { - Expertengruppe 14 der EDQM, European Pharmacopoeia } \\
\text { - Clinical trial task force der EANM } \\
\text { - DFG (Teilnahme an Expertengesprächen zu apparativer Ausstattung der deutschen Nuklearmedizin und zur } \\
\text { - Radionuklidversorgung: Profes. B. Neumaier/M. Patt/H. J. Wester) } \\
\text { - Anderweitige Aktivitäten (z. B. Studien, Publikationen) } \\
\text { - Umfrage zur rechtlichen Grundlage von selbst hergestellten Radiopharmaka } \\
\text { - Initiative zur Durchführung von gemeinsamen Audits bei Herstellern von Ausgangsstoffen und ext. Prüflabors } \\
\text { - Kommentierung des neuen Annex 1 des EU GMP Leitfadens (in Kooperation mit EANM RPC) }\end{array}$ \\
\hline
\end{tabular}


- Tab. 1 Fortsetzung

\section{\begin{tabular}{l|l} 
Ausschuss, $\quad$ Zusammenkünfte, Aktivitäten im Berichtszeitraum und Planungen \\
\hline
\end{tabular} \\ Vorsitz}

\section{Radiopharmaka Vorhaben} (Fortsetzung)
- GMP-Schulung anlässlich AGRR-Jahrestagung, Herbst 2019

- Ausschusssitzung anlässlich DGN-Jahrestagung, 03.04.2019

- Ausschusssitzung anlässlich AGRR-Jahrestagung, Herbst 2019

- Harmonisierung der Aktivitäten der Arbeitsgruppe zu gemeinsamen Lieferanten-Audits mit der europäischen Initiative (SASAI der EANM)

- Schulungsveranstaltung für Ärzte, die unter §13 Abs. 2b des AMG selber Radiopharmaka herstellen, anlässlich DGN-Jahrestagung in Bremen Schilddrüse,
Prof. Dr. Dr. F. Verburg

\begin{tabular}{l} 
Veranstaltungen \\
\hline Aktivitäten
\end{tabular}

- Sitzung im Rahmen der DGN-Jahrestagung, 18.04.2018, Bremen

- Leitlinien

- Diagnostische Ganzkörperszintigraphie, Version 5

- Fort- und Weiterbildungsaktivitäten

- Tagung des Ausschusses Schilddrüse, 09.-10.11.2018, Würzburg

- Fortbildung bei der DGN-Jahrestagung in Bremen

- Beteiligung an DGN-Veranstaltungen oder anderen Veranstaltungen

- DGN-Jahrestagung in Bremen

- Eigene Veranstaltungen

- Tagung des Ausschusses Schilddrüse, 09.-10.11.2018, Würzburg

- Zusammenarbeit mit DGN-Gremien

- Gemeinsame Arbeit mit dem Ausschuss Leitlinien an der S3-Leitlinie „Schilddrüsenkarzinom“

- Zusammenarbeit mit anderen Fachgesellschaften

- Deutsche Gesellschaft für Endokrinologie, Sektion Schilddrüse

- CAEK

- Zusammenarbeit mit anderen Gremien/Kommissionen

- DIN-Norm prätherapeutische Dosimetrie bis Radioiodtherapie gutartiger Schilddrüsenerkrankungen

Strahlenschutz, Veranstaltungen

- Sitzung im Rahmen der DGN-Jahrestagung, 18.04.2018, Bremen

- Strahlenschutzverordnung, Verbändeanhörung; Orientierungsleitfaden SSK; Entlassungsdosis bei Therapie; Stellungnahme kutane Therapie mit Re-188; Augenlinsendosis; GAPTN-Tagung; DIN-NAR Info; ZAES Info

Burchert
- Ausschuss-Sitzung, 16.11.2018, Berlin

- Bericht Anhörung Strahlenschutzverordnung; aktueller Stand; Bericht generische Rechtfertigung FG im BfS; Perspektive Strahlenschutzverordnung; Planungen RL Strahlenschutz 2019; Planungen Fachkunderichtlinie im Strahlenschutz; Medizinische Forschung im neuen Strahlenschutzrecht

Aktivitäten

- Leitlinien, Gesetze

- Kommentierung des Gesetzentwurfs im Rahmen der Verbändeanhörung der neuen Strahlenschutzverordnung; TKs

- Treffen AG Kommentierung StrSchV, 19.06.2018, Hannover; Erstellung einer schriftlichen Stellungnahme

- Verbändeanhörung des BMU zum Gesetzentwurf Strahlenschutzverordnung. 16.07.2018, BMU, Bonn

- Fort- und Weiterbildungsaktivitäten

- GAPTN; 02.-03.02.2018, Medizinische Hochschule Hannover

- Beteiligung an DGN-Veranstaltungen oder anderen Veranstaltungen

- DGN-Jahrestagung in Bremen, Ausschuss-Sitzung, Berlin s. o.

- BfS-Fachgespräch: Rechtfertigung von Tätigkeitsarten, 07.08.2018; BfS Oberschleißheim. Dazu DGN-TK.

- BfS-Konsultationstreffen: Medizinische Forschung. 22.10.2018; BfS Oberschleißheim

- Jahrestreffen der Ethikkommissionen Deutschlands. Forschung: Vortrag „Medizinische Forschung im neuen Strahlenschutzrecht aus Sicht der EK“; 08.11.2018, Berlin

- Zusammenarbeit mit DGN-Gremien

- Arbeitsgemeinschaft GAPTN

- Ausschuss Leitlinien

- Ausschuss Therapie

- Vorstand DGN

- Ausschuss Dosimetrie

- Ausschuss Medizinische Physik

- Zusammenarbeit mit anderen Fachgesellschaften

- EANM Dosimetry Commitee

- EANM Physics Commitee

- DEGRO

- DRG

- DGMP 
- Tab. 1 Fortsetzung

\begin{tabular}{|c|c|c|}
\hline & \multicolumn{2}{|c|}{ Zusammenkünfte, Aktivitäten im Berichtszeitraum und Planungen } \\
\hline \multirow[t]{2}{*}{$\begin{array}{l}\text { Strahlenschutz, } \\
\text { (Fortsetzung) }\end{array}$} & $\begin{array}{l}\text { Aktivitäten } \\
\text { (Fortsetzung) }\end{array}$ & $\begin{array}{l}\text { - Zusammenarbeit mit anderen Gremien/Kommissionen } \\
\text { - Strahlenschutzkommission (SSK) } \\
\text { - Normenausschuss Radiologie (NAR des Deutschen Instituts für Normung e. V. (DIN). } \\
\text { - Bundesamt für Strahlenschutz (u. a. Fachgespräche, Gesetzesvorbereitung) } \\
\text { - Bundesministerium für Umwelt, Naturschutz, Bau und Reaktorsicherheit (BMUB) } \\
\text { - Anderweitige Aktivitäten (z. B. Studien, Publikationen) } \\
\text { - Stellungnahmen zu GBA-Entschlüssen s. o. } \\
\text { - Ausführliche Stellungnahme der DGN im Rahmen der Verbändeanhörung zur Strahlenschutzverordnung }\end{array}$ \\
\hline & Vorhaben & $\begin{array}{l}\text { - Kommentierung Orientierungsleitfaden SSK; 2. Quartal } 2019 \\
\text { - Strahlenschutzgesetzgebung: Richtlinie Strahlenschutz in der Medizin; Fachkunderichtlinien; Ende } 2019 \\
\text { - E-Learning Handhabungen Strahlenschutzgesetz; } 2019 \\
\text { - Einführung von Team-Chat (Slack); 3. Quartal } 2019\end{array}$ \\
\hline \multirow[t]{2}{*}{$\begin{array}{l}\text { Therapie, } \\
\text { Dr. T. Pöppel }\end{array}$} & Veranstaltungen & $\begin{array}{l}\text { - Sitzung im Rahmen der DGN-Jahrestagung 2018, 18.4.18, Bremen } \\
\text { - Ausschuss-Sitzung, 16.11.18, Berlin }\end{array}$ \\
\hline & Aktivitäten & $\begin{array}{l}\text { - Leitlinien } \\
\text { - Initiierung MIBG-Therapie-Leitlinie } \\
\text { - Erstellung 3-Länder-Leitlinie RSO (OGN, DGN, SGNM) } \\
\text { - Initiierung Aktualisierung Ra-223-Therapie-Leitlinie } \\
\text { - Erstellung FDG-PET/CT-Leitlinie } \\
\text { - Beteiligung an DGN-Veranstaltungen oder anderen Veranstaltungen } \\
\text { - Mitgestaltung DGN-Sitzungen auf dem 33. Deutsche Krebskongress 2018, Berlin } \\
\text { - Organisation Therapieweiterbildungssitzung auf der DGN-Jahrestagung 2019 } \\
\text { - Zusammenarbeit mit DGN-Gremien } \\
\text { - DRG und Leistungserfassung, Leitlinien, Fort- und Weiterbildung, Strahlenschutz, PET } \\
\text { - Zusammenarbeit mit anderen Fachgesellschaften } \\
\text { - DGE } \\
\text { - Zusammenarbeit mit anderen Gremien/Kommissionen } \\
\text { - NET-Register } \\
\text { - Nuderweitige Aktivitäten (z. B. Studien, Publikationen) }\end{array}$ \\
\hline
\end{tabular}

- Tab. 2 Jahresberichte 2018: DGN-Arbeitsgemeinschaften

Arbeitsgemeinschaf, Vorsitz

In-vitro Diagnostik, PD Dr. M. Zimny

\section{Zusammenkünfte, Aktivitäten im Berichtszeitraum und Planungen}

Veranstaltungen • Sitzung im Rahmen der DGN-Jahrestagung, 20.04.2018, Bremen

Aktivitäten $\quad$ - Beteiligung an DGN-Veranstaltungen oder anderen Veranstaltungen

- DGN-Jahrestagung in Bremen

- Zusammenarbeit mit anderen Gremien/Kommissionen

- Berufsverband Deutscher Nuklearmediziner (BDN)

Vorhaben - Sicherstellung der Vermittlung der Weiterbildungsinhalte „in-vitro-Diagnostik“ im Rahmen der nuklearmedizinischen Facharztausbildung

\section{Gemeinsamen}

Arbeitskreises Physik

und Technik

in der Nuklear-

medizin, (GAPTN),

Prof. Dr. W. Burchert

- Ausschuss Strahlenschutz
- Sitzung im Rahmen der DGN-Jahrestagung, 19.04.2018, Bremen

- 16. GAPTN Jahrestreffen, 02.-03.02.2018, Medizinische Hochschule Hannover, Hannover

\begin{tabular}{l|l} 
Aktivitäten & Zusammenarbeit mit DGN-Gremien
\end{tabular}

- Ausschuss Medizinphysik

- Zusammenarbeit mit anderen Fachgesellschaften - DGMP

- Zusammenarbeit mit anderen Gremien/Kommissionen)

- NAR-DIN

- Strahlenkommission (A2, A4)

\begin{tabular}{l|ll} 
Vorhaben & Sitzung im Rahmen der DGN-Jahrestagung, 2019, Bremen
\end{tabular}

- 17. GAPTN Jahrestreffen 2020, Hannover 
> Tab. 2 Fortsetzung

Arbeitsgemeinschaf, Vorsitz

Kardiovaskuläre Nuklearmedizin und Lungendiagnostik, Prof. Dr. Dr. W. Schäfer
Zusammenkünfte, Aktivitäten im Berichtszeitraum und Planungen

\author{
Veranstaltungen • Sitzung im Rahmen der DGN-Jahrestagung, 20.04.2018, Bremen \\ \begin{tabular}{l|l} 
Aktivitäten $\quad$ Leitlinien \\
\hline
\end{tabular} \\ - Neue S1 Leitlinie zur Lungenszintigraphie der DGN wurde publiziert
}

- Fort- und Weiterbildungsaktivitäten

- 22.-23.02.2018, 13. Kurs für kardiovaskuläre Nuklearmedizin/Nuklearkardiologie, Garmisch-Partenkirchen

- Beteiligung an DGN-Veranstaltungen oder anderen Veranstaltungen

- Fortbildungsvortrag im Rahmen des Deutschen Röntgen-Kongress (09.05.2018, Das Herz: Wenn PET, wann SPECT, wann MRT?)

- Fortbildungsvortrag im Rahmen des European Congress of Radiology (02.03.2018, Cardiac hybrid imaging indications: case-based)

- Eigene Veranstaltungen

- Sitzung der Arbeitsgruppe „Nuklearkardiologische Diagnostik“, 04.04.2017, Mannheim im Rahmen der DGK

- Zusammenarbeit mit anderen Fachgesellschaften

- DGK

- Positionspapier Nuklearkardiologie - Update 2018 mit DGK, Publikation in der Nuklearmedizin und im Kardiologen

- BDN

- EANM

- Zusammenarbeit mit anderen Gremien/Kommissionen

- Mitglied des Cardiovaskular Committee der EANM

- Beteiligung an der ESMIT Summer School in Belgrad

- Anderweitige Aktivitäten (z. B. Studien, Publikationen)

- Policy paper nuclear cardiology - update 2018 - Current status of clinical practice. Prof. O. Lindner, J. Bauersachs, F. M. Bengel, W. Burcher, J. Vom Dahl, R. Dörr, M. Hacker, M. Kelm, T. Rassaf, C. Rischpler, W. Schäfer, M. Schäfers, S. Silber, R. Zimmermann. Nuklearmedizin. 2018;57:146-152.

Vorhaben $\quad$ • 14. Kurs für kardiovaskuläre Nuklearmedizin/Nuklearkardiologie, 12.-14.09.2019, Potsdam

- Nuklearkardiologie Workshop bei den Kardiodiagnostiktagen 2019 (21.-23.02.19), Lindner/Bucerius

- 8. Umfrage zur Myokard-SPECT (Start Januar 2019)

Medizinisch-tech-

Veranstaltungen

- Sitzung im Rahmen der DGN-Jahrestagung, 20.04.2018, Bremen

- Mitgliederversammlung im Rahmen der DGN-Jahrestagung, 20.04.2018, Bremen

- Vorstandssitzung, 03.-04.08.2018, Heidelberg

- Diverse Telefonkonferenzen

Aktivitäten $\quad$ - Fort- und Weiterbildungsaktivitäten

- Organisation der MTRA-Tagung im Rahmen der DGN-Jahrestagung in Bremen

- Beteiligung an DGN-Veranstaltungen oder anderen Veranstaltungen

- Durchführung von Seminaren in Zusammenarbeit mit dem DVTA

- Durchführung eines vom DIW zertifizierten Workshops auf der Jahrestagung in Bremen

- Zusammenarbeit mit anderen Fachgesellschaften

- Erfahrungsaustausch mit VMTB und DVTA über Problematik des Fachkräftemangels

- Erarbeiten der Stellungnahme zum Positionspapier des VMTB zu dieser Thematik für den Vorstand der DGN

\begin{tabular}{l|l} 
Vorhaben & - Vorbereitung und Organisation der MTRA-Tagung im Rahmen der DGN-Jahrestagung 2019, Bremen
\end{tabular}

- Weiterbildungsmöglichkeiten mittels neuer Medien, seit 7. 2017

Neuronuklear-

medizin,

Prof. Dr. K.-J. Langen
- Sitzung im Rahmen der DGN-Jahrestagung, 19.04.2018, Bremen

- Leitlinien

- S1- Leitlinie für SPECT-Untersuchungen mit dem 123I-markierten Dopamintransporter-Liganden FPCIT (DaTSCANTM) ist seit April 2018 bei der AWMF online

- S1-Leitlinie Hirnperfusions-SPECT mit Tc-99 m Radiopharmaka wird derzeit aktualisiert

Vorhaben
- Publikation der S1- Leitlinie für „SPECT- Untersuchungen mit dem 123I-markierten Dopamintransporter-Liganden FP-CIT (DaTSCANTM)“ in der Zeitschrift Nuklearmedizin, 2018/2019

- Erstellung einer neuen Leitlinie Hirntumordiagnostik, bis Ende 2019 
- Tab. 2 Fortsetzung

\section{Arbeitsgemeinschaf, Vorsitz}

Pädiatrische Nuklearmedizin,

Prof. Dr. C. Franzius

\section{Zusammenkünfte, Aktivitäten im Berichtszeitraum und Planungen}

Veranstaltungen

Aktivitäten

- Sitzung im Rahmen der DGN-Jahrestagung, 21.04.2018, Bremen

- Leitlinien

- Leitlinie „MIBG-Szintigraphie bei Kindern“ Durchsicht/Aktualisierung

- Leitlinie „Schilddrüsenkarzinom bei Kindern“ Durchsicht/Aktualisierung

- Es ist 2018 eine neue gemeinsame Leitlinie der SNMMI/EANM erarbeitet worden: „SNMMI Procedure Standard/EANM Practice Guideline for Diuretic Renal Scintigraphy in Adults With Suspected Upper Urinary Tract Obstruction 1.0". Hier erfolgt derzeit die Überarbeitung und Anpassung der DGN-Leitlinie „Nierenfunktionsszintigraphie“ an diese Guideline.

- Aktualisierung der Leitlinie „PET im Kindesalter“ ist noch nicht abgeschlossen. Diese soll um einen Kommentar zur PET/MRT ergänzt werden.

- Alle anderen pädiatrisch-nuklearmedizinischen AWMF-Leitlinien liegen aktualisiert vor, nächste Aktualisierungen 2019-2020

- Zusammenarbeit mit DGN-Gremien

- Ausschuss Klinisches Qualitätsmanagement/Leitlinien

- Zusammenarbeit mit anderen Fachgesellschaften

- Gesellschaft für Pädiatrische Hämatologie und Onkologie (GPOH):

- Kontakt zu Leitlinienkoordinatorin zwecks Mitarbeit an Aktualisierung der GPOH-Leitlinien (Prof. Dr. U. Creutzig).

- Diskussion und Erfahrungsaustausch zur Teilnahme an multizentrischen Studien der GPOH:

- Neuroblastomstudie (Prof. Dr. M. Schmidt), neue Leitung der GPOH Studie Frau Prof. Eggert, Charité, Berlin, Diskussion über die F-18-DOPA-PET bei Neuroblastom-Patienten

- Hodgkin-Studie EuroNet-PHL (Prof. Dr. R. Kluge):

- C2-Protokoll. Standardisierung der PET/MRT erwünscht (wird derzeit in 12 Zentren in Europa durchgeführt), Fragebogenabfrage ist erfolgt

- FET-PET (Prof. Dr. M. Plotkin): Zusammenarbeit mit AG Nuklearmedizin und Strahlentherapie der DGN und DEGRO: GLIAA-Studie, FET-PET-Studie wurde Ende 2018 geschlossen

- Zusammenarbeit mit anderen Gremien/Kommissionen

- Enge Zusammenarbeit mit dem Paediatric Committee der EANM (L. Kurch)

- Dosimetry Committee der EANM (über Prof. Dr. M. Lassmann) Anpassung FDG-Aktivitäten im Kindesalter, Einbringung der Erfahrungen aus Deutschland mit pädiatrischen PET/MRT-Untersuchungen

Vorhaben - Abschluss der Aktualisierung der o. g. Leitlinien „PET im Kindesalter“ und „Nierenfunktionsszintigraphie“, 2019

Radiochemie, Radiopharmazie, Prof. Dr. K. Kopka
Veranstaltungen

- 26. Jahrestagung der AGRR (AGRR2018) (208 TeilnehmerInnen), 20.-22.09.2018, Aachen

- 26. Mitgliederversammlung, 21.09.2018, Aachen

- 57. Komitee-Sitzung, 20.09.2018, Aachen

- 56. Komitee-Sitzung, 20.04.2018, Bremen

- 55. Komitee-Sitzung, 16.03.2018, Aachen

Aktivitäten

- Leitlinien
- Leitlinien der AGRR gültig seit 25.09.2009

- Fort- und Weiterbildungsaktivitäten

- Vierter AGRR-GMP Workshop am 20.09.2018, Aachen: R. Schulze (Dresden), Datenintegrität

- S. Gärtner (Bad Bocklet), Radiopharmaka - Prüfung auf Sterilität und Endotoxine

- Plenarvorträge im Rahmen der 26. AGRR-Tagung als Bestandteil des Programms: Prof. Dr. med. F. Kießling, University of Aachen: Multiparametrische Diagnostik und Theranostik von Tumoren

Prof. Dr. rer. nat. B. Neumaier, Jülich: Einfluss neuer Radiofluorierungsmethoden auf die Entwicklung der präklinischen und klinischen PET-Bildgebung

Dr. K. Gagnon, Uppsala, Sweden: Liquid targets beyond ${ }^{18} \mathrm{~F}$ and ${ }^{13} \mathrm{~N}$ for the production of radionuclides by medical cyclotrons

Dr. D. van der Born, Nijmegen, The Netherlands: Microfluidic flow technology: Can it revolutionize the production of radiopharmaceuticals?

- Fachgruppe Nuklearchemie der GDCh (Beirat-Vertreter Prof. B. Neumaier)

- Postgraduate Certificate Course in Radiopharmaceutical Chemistry/Radiopharmacy (EANM) 
- Tab. 2 Fortsetzung

Arbeitsgemeinschaf, Vorsitz

Radiochemie, Radiopharmazie, (Fortsetzung)
Zusammenkünfte, Aktivitäten im Berichtszeitraum und Planungen

Aktivitäten $\quad$ - Beteiligung an DGN-Veranstaltungen oder anderen Veranstaltungen

(Fortsetzung)

- Tagung des DGN-Ausschusses „Hochschulfragen“, 16.-17.11.2018, Berlin

- Vortrags- und Posterprogramm im Rahmen des 30th Annual Congress of the European Association of Nuclear Medicine (EANM'18), 13.-17.10.2018, Düsseldorf

- Vortrags- und Posterprogramm im Rahmen des 3 rd International Edition of the Symposium on Technetium and Other Radiometals in Chemistry and Medicine (TERACHEM 2018), 26.-29.09.2018, Bressanone, BZ, Italien

- Vortrags- und Posterprogramm im Rahmen des 65th Annual Meeting der Society of Nuclear Medicine and Molecular Imaging (SNMMI), 23.-26.06.2018, Philadelphia, PA, USA

- Tagung des DGN-Ausschusses „Hochschulfragen“, 21.-22.04.2018, Bremen

- Vortrags- und Posterprogramm im Rahmen der Jahrestagung der DGN (NuklearMedizin 2018), 18.-21.04.2018, Bremen

- Eigene Veranstaltungen

- 26. Jahrestagung der AGRR (AGRR2018), 20.-22.09.2018, Aachen

- Vierter AGRR-GMP Workshop, 20.09.2018, Aachen (GMP-Beauftragter Dr. Christoph Solbach, Ulm)

- Zusammenarbeit mit DGN-Gremien

- DGN Ausschuss Hochschulfragen

- DGN Radiopharmaka-Ausschuss

- DGN Ausschuss Multicenterstudien (beratend)

- Zusammenarbeit mit anderen Fachgesellschaften

- Society of Radiopharmaceutical Sciences (SRS)

- European Association of Nuclear Medicine (EANM)

- Fachgruppe Nuklearchemie der Gesellschaft Deutscher Chemiker (GDCh)

- Deutsche Pharmazeutische Gesellschaft (DPhG)

- ETH Postgraduate Course Certificate of Advanced Studies (CAS) in Radiopharmaceutical Chemistry/ Radiopharmacy, recognised by EANM

- Österreichische Gesellschaft für Nuklearmedizin und Molekulare Bildgebung (OGNMB)

- Bündnis zur Förderung der Radiopharmazeutischen Wissenschaften (BüFraWi)

- Zusammenarbeit mit anderen Gremien/Kommissionen

- EANM Radiopharmacy Committee (Prof. Dr. Marianne Patt, Leipzig, Mitglied seit 01.01.2015)

- EANM Drug Development Committee (Dr. Winnie Deuther-Conrad, Leipzig, Mitglied seit 01.01.2016)

- Zentralstelle der Länder für Gesundheitsschutz bei Arzneimitteln und Medizinprodukten (ZLG), EFG (Expertenfachgruppe) 12 - Radiopharmaka (Leitung: Dr. Michaela Karmann), Klärung von aktuellen Fragen des GMP/Arzneimittelrechts

- European Directorate for the Quality of Medicines (edqm) - Expertengruppe 14 der Europäischen Arzneibuchkommission

- BfArM - AG Radiopharmaka

- Anderweitige Aktivitäten (z. B. Studien, Publikationen)

- AGRR-Leitlinien

Deutsches Konsortium für Translationale Krebsforschung (DKTK):

- Radiopharmazie für die klinische Prüfung der Phasen-I und -II mit dem Radiopharmakon 68GaPSMA-11

- Vergabe des AGRR-Preises für die besten Vorträge 2018 (Nachwuchswissenschaftler):

- Thomas Lindner (Postdoktorand, Universitätsklinikum Heidelberg): Entwicklung von Fibroblasten-Aktivierungsprotein alpha bindender Tracer für den Einsatz in Diagnostik und Therapie

- F. Reissig (Doktorand, HZDR, AG Constantin Mamat): Chelatoren für die Komplexierung schwerer Erdalkalimetallionen

- H. Ahmed (Doktorand, ETH Zürich, AG Simon Ametamey): Evaluation of Carbon-11 and Fluorine-18 Labeled Benzazepine Analogues For Imaging the GluN2B Subunit of the N-Methyl-D-Aspartate Receptor"

Vorhaben • 27. Jahrestagung der AGRR (AGRR2019), 12.-14.09.2019, Pamhagen, Österreich (Markus Mitterhauser, Wolfgang Wadsak

- 28. Jahrestagung der AGRR (AGRR2020), 2020, Wittenberg (M. Patt, P. Brust)

- 29. Jahrestagung der AGRR (AGRR2021), angefragt: Tübingen

- 30. Jahrestagung der AGRR (AGRR2022), in Planung 
- Tab. 3 Jahresberichte 2018: Regionalgesellschaften

\section{Regionalgesellschaft, Vorsitz}

Berlin-Brandenburgische Gesellschaft für Nuklearmedizin, Dr. G. J. Förster

\section{Zusammenkünfte, Aktivitäten im Berichtszeitraum und Planungen}

Veranstaltungen $\quad$ - 1. Vorstandssitzung, 28.02.2018, Berlin

- 2. Vorstandssitzung, 20.04.2018, Bremen

- Vollversammlung 2018 nach $\S 10$ der Satzung der BBGN, 04.07.2018, Berlin

- 3. Vorstandssitzung, 21.08.2018, Berlin

- 4. Vorstandssitzung, Telefonkonferenz, 31.10.2018

- Eigene Veranstaltungen

- MTRA-Fortbildung 2018, 26.09.2018, Konferenzzentrum, Potsdam Programm:

- Myokard-SPECT

- Notfallmanagement bei Belastungsuntersuchungen

- Mitgliederversammlung 2018, Seminaris CampusHotel, 09.11.2018, Berlin

- Jahrestagung 2018, Seminaris CampusHotel, 10.11.2018, Berlin Programm:

- Neue Techniken und Therapieansätze in der Nuklearmedizin

- Nicht-thyreoidale Themen in der Schilddrüsensprechstunde

\section{Bayerische Gesell-} schaft für NuklearVeranstaltungen medizin,

Prof. Dr. J. Sciuk

- 39. Jahrestagung der BGN, 06./07.07.2018, Nürnberg

- Thematische Schwerpunkte:

Wissenschaftliche Vorträge aus den Universitätsklinika Demenz: Klinik, Neuropathologie, PET/SPECT Onkologie: OPTIMAL > 60-Studie, SPECT bei Prostatakarzinom, CXCR4, personalisierte Medizin Fortbildung

Lungenembolie, Schilddrüse und Nebenschilddrüse

\begin{tabular}{l|l} 
Aktivitäten & $\bullet$ Zusammenarbeit mit DGN-Gremien
\end{tabular}

- Zusammenarbeit mit dem DGN-Vorstand und dem Hochschulausschuss an der MWBO-Novelle

- Fort- und Weiterbildungsaktivitäten

- MTRA-Tagung anlässlich der Jahrestagung der BGN in Nürnberg am 06.07.2018:

- Radiochemie, Radioiodtherapie, SLNE, Myokard-SPECT, Hirn-SPECT

- Regelmäßiger Fortbildungszirkel der MTRA aus den bayerischen Großklinika

- Aktualisierungskurse nach StrISchv

- Beteiligung an DGN-Veranstaltungen oder anderen Veranstaltungen

- Fortbildungsvortrag im Rahmen des Deutschen Röntgen-Kongress (09.05.2018, Das Herz: Wann PET, wann SPECT, wann MRT?)

- Fortbildungsvortrag im Rahmen des European Congress of Radiology (02.03.2018, Cardiac hybrid imaging indications: case-based)

- Eigene Veranstaltungen

- Sitzung der Arbeitsgruppe „Nuklearkardiologische Diagnostik“, 04.04.2017, Mannheim im Rahmen der DGK

- Zusammenarbeit mit anderen Fachgesellschaften

- Bayerische Röntgengesellschaft

- Zusammenarbeit mit anderen Gremien/Kommissionen

- Regionalsektion Süd-Ost und Franken der DGMP

- Landesverband Bayern des Berufsverbands Deutscher Nuklearmediziner

- Bayerische Landesärztekammer

- Ärztliche Stelle der BLAEK

- Landesamt für Umweltschutz

- Anderweitige Aktivitäten (z. B. Studien, Publikationen)

- Wolfgang-Becker-Forschungspreis der BGN MTRA-Preis der BGN

\begin{tabular}{l|ll} 
Vorhaben & - 40. Jahrestagung der BGN, 05./06.07.2019, Würzburg
\end{tabular}

Veranstaltungen • Vorstandssitzung, 20.04.2018, Bremen

- 28. Jahrestagung der GNS (Tagungspräsidenten Dr. A. Odparlik, Dr. J. Grimm), 15.-16.06.2018, Leopoldina, Halle/Saale

- Vorstandssitzung, 15.06.2018, Halle/Saale

- Mitgliederversammlung, 15.06.2018, Halle/Saale 
- Tab. 3 Fortsetzung

\begin{tabular}{|c|c|c|}
\hline Regionalgesellschaft, & \multicolumn{2}{|c|}{ Zusammenkünfte, Aktivitäten im Berichtszeitraum und Planungen } \\
\hline \multirow[t]{2}{*}{$\begin{array}{l}\text { Gesellschaft für } \\
\text { Nuklearmedizin } \\
\text { Sachsen, } \\
\text { (Fortsetzung) }\end{array}$} & Aktivitäten & $\begin{array}{l}\text { - Fort- und Weiterbildungsaktivitäten } \\
\text { - 43. Leipziger Fortbildung für Ärzte und MTRA, 11.04.2018, InterCityHotel Leipzig, Zyklus „Aus der } \\
\text { Praxis - für die Praxis“ } \\
\text { Thema: Lungen V/P SPECT-CT, State of the Art } 2018 \\
\text { - Dresdner Fortbildung für Ärzte und MTRA, Universitätsklinikum TU Dresden, Hörsaal Haus } 19 \\
\text { Thema: Nuklearmedizinische Nierendiagnostik aus der Sicht des Urologen / Nierensequenzszintigra- } \\
\text { phie - state of the art / Nuklearmedizinische Methoden der Clearance-Bestimmung } \\
\text { - } 44 . \text { Leipziger Fortbildung für Ärzte und MTRA, 07.11.2018, InterCityHotel Leipzig } \\
\text { Themen: Technische Grundlagen PET/CT / Qualitätskontrolle von Radionukliden } \\
\text { - Beteiligung an DGN-Veranstaltungen oder anderen Veranstaltungen } \\
\text { - DGN-Mitgliederversammlung, 20.04.2018, Messe Bremen } \\
\text { - NET-Patiententag, 27.10.2018, DZNE Haus 64, Magdeburg } \\
\text { - Eigene Veranstaltungen } \\
\text { - Arbeitstreffen ,MTRA Fortbildung für Ärzte und MTRA`s (Stammtisch)` der Gesellschaft für Nuklear- } \\
\text { - medizin Sachsen e. V. (GNS), 24.01.2018, InterCityHotel Leipzig } \\
\text { - Zusammenarbeit mit DGN-Gremien } \\
\text { - diverse Ausschüsse der DGN } \\
\text { - Zusammenarbeit mit anderen Fachgesellschaften } \\
\text { - Sächsische Röntgengesellschaft (SRG) e. V. }\end{array}$ \\
\hline & Vorhaben & $\begin{array}{l}\text { - 29. Jahrestagung der GNS - gemeinsame Tagung mit der Norddeutschen Gesellschaft für Nuklear- } \\
\text { medizin (NGN) e. V. (800 Jahre Rostock/600 Jahre Universität Rostock) } \\
\text { - Beteiligung an der gemeinsamen Jahrestagung der SRG und Thüringischen } \\
\text { Gesellschaft für Radiologie und Nuklearmedizin (TGRN) e. V., 30.08.-01.09.2019, Oberwiesenthal }\end{array}$ \\
\hline \multirow[t]{3}{*}{$\begin{array}{l}\text { Mittelrheinische } \\
\text { Gesellschaft für } \\
\text { Nuklearmedizin, } \\
\text { Prof. Dr. M. Schrecken- } \\
\text { berger }\end{array}$} & Veranstaltungen & $\begin{array}{l}\text { - Jahrestagung 2018, } 10.11 .2018 \\
\text { - Tagungshotel Zugbrücke Grenzau, Höhr-Grenzhausen } \\
\text { - Tagungspräsident: Prof. Dr. med. H. Wieler, Klinik für Nuklearmedizin BWZK Koblenz } \\
\text { - Verleihung des Dagmar-Eißner-Preises an Dr. M. A. Hoffmann, Koblenz und an Herrn Dr. R. Werner, } \\
\text { - Uniklinik Würzburg } \\
\text { - Verleihung des Thomas-Behr-Gedächtnis-Preises an E. Riehl, Uniklinik Marburg } \\
\text { - Mitgliederversammlung, 10.11.2018, Tagungshotel Zugbrücke Grenzau, Höhr-Grenzhausen }\end{array}$ \\
\hline & Aktivitäten & $\begin{array}{l}\text { - Fort- und Weiterbildungsaktivitäten } \\
\text { - Strahlenschutzkurse zur Aktualisierung der Fachkunde im Strahlenschutz für Ärzte, MTA, MTRA und } \\
\text { MPE 11/2018 (Leitung Prof. Dr. H. Wieler, BWZK Koblenz) } \\
\text { - Zusammenarbeit mit anderen Gremien/Kommissionen } \\
\text { - Landesärztekammer Rheinland/Pfalz }\end{array}$ \\
\hline & Vorhaben & $\begin{array}{l}\text { - Jahrestagung 2019, 09.11.2019, Klinikum Fulda } \\
\text { - Kongresspräsident: PD Dr. A. Hertel, 04/2018, Bremen }\end{array}$ \\
\hline \multirow[t]{2}{*}{$\begin{array}{l}\text { Norddeutsche Gesell- } \\
\text { schaft für Nuklear- } \\
\text { medizin, } \\
\text { Prof. Dr. V. Barsegian }\end{array}$} & Veranstaltungen & $\begin{array}{l}\text { - 23. Rostocker Schilddrüsensymposium, } 10.03 .2018 \text {, Rostock } \\
\text { - 7. MTRA Stammtisch, 21.03.2018, Hamburg } \\
\text { - 29. Jahrestagung der NGN, 01. und 02.06.2018, Schwerin } \\
\text { - 25. Hamburger Nuklearmedizin-Tage, 13.-15.06.2018 } \\
\text { - XXV. Kieler Schilddrüsensymposium, 08.09.2018, Kiel } \\
\text { - } 48 . \text { Regionaltagung Nuklearmedizin MV, } 05.10 .2018 \text {, Güstrow } \\
\text { - } 8 \text { MTRA Stammtisch, } 24.10 .2018 \text {, Hamburg }\end{array}$ \\
\hline & Aktivitäten & $\begin{array}{l}\text { - Fort- und Weiterbildungsaktivitäten } \\
\text { - 25. Hamburger Nuklearmedizin-Tage 13.-15.06.2018 } \\
\text { - 48. Regionaltagung Nuklearmedizin MV, 05.10.2018, Güstrow } \\
\text { - 7. MTRA Stammtisch, 21.03.2018, Hamburg } \\
\text { - 8. MTRA Stammtisch, 24.10.2018, Hamburg } \\
\text { - 23. Rostocker Schilddrüsensymposium, 10.03.2018, Rostock } \\
\text { - XXV. Kieler Schilddrüsensymposium, 08.09.2018, Kiel } \\
\text { - Eigene Veranstaltungen } \\
\text { - 29. Jahrestagung der NGN, 01. und 02.06.2018, Schwerin } \\
\text { - Zusammenarbeit mit DGN-Gremien } \\
\text { - Ausschuss PET } \\
\text { - Zusammenarbeit mit anderen Fachgesellschaften } \\
\text { - Muskuloskelettaler Arbeitskreis Hämophilie } \\
\text { - Anderweitige Aktivitäten (z. B. Studien, Publikationen) } \\
\text { - Mitarbeit Pschyrembel: Fachbereich Nuklearmedizin und zugehörige Physik, Medizintechnik und } \\
\quad \text { Radiopharmazie; } \\
\quad \text { Printausgabe vervollständigt, Online-Ausgabe in Bearbeitung }\end{array}$ \\
\hline
\end{tabular}


- Tab. 3 Fortsetzung

\section{Regionalgesellschaft, Vorsitz}

Norddeutsche Gesell- Vorhaben schaft für Nuklearmedizin

(Fortsetzung)

Rheinisch-West-

fälische Gesellschaft für Nuklearmedizin, Prof. Dr. Dr. W. Schäfer

\section{Zusammenkünfte, Aktivitäten im Berichtszeitraum und Planungen}

- 30. Jahrestagung der NGN gemeinsam mit der Gesellschaft für Nuklearmedizin Sachsen am 14.06.2019 in Rostock

- 24. Rostocker Schilddrüsensymposium, 16.03.2019, Rostock

- Hamburger Nuklearmedizin-Tage 05.-07.06.2019

- 49. Regionaltagung Nuklearmedizin MV, Ende 2019, Schwerin

- Schweriner Nuklearmedizin Tage, Ende 2019, Schwerin

Veranstaltungen $\quad$ • 45. Jahrestagung der RWGN, Tagungspräsident Prof. Dr. R. Larisch, 30.11.-01.12.2018, Lüdenscheid

\begin{tabular}{l|l} 
Aktivitäten & - Fort- und Weiterbildungsaktivitäten
\end{tabular}

- MTRA-Fortbildung im Rahmen der 45. Jahrestagung der RWGN

- Zusammenarbeit mit DGN-Gremien

- Arbeitsgemeinschaft Kardiovaskuläre Nuklearmedizin und Lungendiagnsotik

- Zusammenarbeit mit anderen Fachgesellschaften

- Zusammenarbeit mit dem BDN

- Anderweitige Aktivitäten (z. B. Studien, Publikationen)

- Ehrenmitgliedschaft von Univ.-Prof. Dr. H.-J. Biersack und Univ.-Prof. Dr. H. Schicha

Vorhaben $\quad$ • 46. Jahrestagung der RWGN, Tagungspräsidenten Prof. Dr. H. Palmedo und Dr. H. Brockmann, Bonn

- MTRA-Fortbildung im Rahmen der 46. Jahrestagung der RWGN in Bonn

Südwestdeutsche Gesellschaft für Nuklearmedizin, Prof. Dr. K. Tatsch
- 30. Wissenschaftliche Jahrestagung der SWDGN, 19. und 20.10.2018, Stuttgart, Tagungspräsidentin: Prof. Dr. G. Pöpperl

Schwerpunkte:

- Schilddrüsenknoten/ Medikamenteninduzierte Schilddrüsenerkrankungen

- Traumatologie/Endoprothetik

- Strahlenschutz / Sentinel Lymphknoten

- Onkologie: Nuklearmedizin in S3 Leitlinien, Radioiodtherapie des Schilddrüsenkarzinoms

- Therapie: Update PSMA-Therapie, Monitoring der Therapie von Knochenmetastasen

MTRA-Programm:

- Management von Notfällen

- Qualitätskontrolle von Gammakameras und Radiopharmaka

- Erweiterung des Berufsbildes MTRA durch Akademisierung

- Pitfalls (interaktiv)

\begin{tabular}{l|l} 
Aktivitäten & - Fort- und Weiterbildungsaktivitäten
\end{tabular}

- Sommerfortbildung der SWDGN

- Funktionsszintigraphie des oberen GI-Traktes

- Inhaltsgleiche Veranstaltungen am 04.07.2018, Universitätsklinikum Tübingen und 12.07.2018, Städtischen Klinikum Karlsruhe (Referent: Prof. Dr. K. Tatsch, Karlsruhe)

Vorhaben $\quad$ - Aktualisierungskurs nach StrSchV und RöV bzw. nach aktuellem Strahlenschutz-Recht am 15. und 16.02.2019 im Universitätsklinikum Heidelberg, Referentin: E. Mörl

- Sommerfortbildung 2019 für MTRA (und Ärzte) im Universitätsklinikum Tübingen sowie im Städtischen Klinikum Karlsruhe (Workshop-Format):

Tipps und Kniffe bei SPECT und SPECT/CT

- 31. Jahrestagung der SWDGN, Tagungspräsident: Prof. Dr. A. Beer, 08. und 09.11.2019, Ulm 